\title{
Differentiation of embryonic stem cells into hepatocytes that coexpress coagulation factors VIII and IX
}

\author{
Jun CAO\#, Chang-zhen SHANG\#, Li-hong LÜ, De-chuan QIU, Meng REN, Ya-jin CHEN*, Jun MIN*
}

Department of Hepatobiliary Surgery, The Second Affiliated Hospital of Sun Yat-sen University, Guangzhou 510120, China

\begin{abstract}
Aim: To establish an efficient culture system to support embryonic stem (ES) cell differentiation into hepatocytes that coexpress F-VIII and F-IX.

Methods: Mouse E14 ES cells were cultured in differentiation medium containing sodium butyrate (SB), basic fibroblast growth factor (bFGF), and/or bone morphogenetic protein 4 (BMP4) to induce the differentiation of endoderm cells and hepatic progenitor cells. Hepatocyte growth factor, oncostatin $\mathrm{M}$, and dexamethasone were then used to induce the maturation of ES cell-derived hepatocytes. The mRNA expression levels of endoderm-specific genes and hepatocyte-specific genes, including the levels of F-VIII and F-IX, were detected by RT-PCR and real-time PCR during various stages of differentiation. Protein expression was examined by immunofluorescence and Western blot. At the final stage of differentiation, flow cytometry was performed to determine the percentage of cells coexpressing F-VIII and F-IX, and ELISA was used to detect the levels of F-VIII and F-IX protein secreted into the culture medium.

Results: The expression of endoderm-specific and hepatocyte-specific markers was upregulated to highest level in response to the combination of SB, bFGF, and BMP4. Treatment with the three inducers during hepatic progenitor differentiation significantly enhanced the mRNA and protein levels of F-VIII and F-IX in ES cell-derived hepatocytes. More importantly, F-VIII and F-IX were coexpressed with high efficiency at the final stage of differentiation, and they were also secreted into the culture medium.

Conclusion: We have established a novel in vitro differentiation protocol for ES-derived hepatocytes that coexpress F-VIII and F-IX that may provide a foundation for stem cell replacement therapy for hemophilia.
\end{abstract}

Keywords: coagulation factor VIII; coagulation factor IX; embryonic stem cells; differentiation; hemophilia; sodium butyrate

Acta Pharmacologica Sinica (2010) 31: 1478-1486; doi: 10.1038/aps.2010.100; published online 18 Oct 2010

\section{Introduction}

Hemophilia is a recessive genetic disease characterized by a severe bleeding diathesis caused by lack of expression of coagulation factors VIII and IX (F-VIII and F-IX) ${ }^{[1-3]}$. Replacement therapy is a standard form of treatment, but plasma-derived or recombinant concentrates of F-VIII and F-IX are both expensive and readily degraded in the blood ${ }^{[4,5]}$. Alternatively, some investigators have reported that hepatocyte transplantation can correct hemophilia A in mice, suggesting that cellbased therapy with adult cells may be a useful approach in the treatment of hemophilia ${ }^{[6]}$. However, a common disadvantage of adult cell transplantation is the shortage of donors, which restricts its wider application.

\footnotetext{
\# These authors contributed equally to this study.

* To whom correspondence should be addressed.

E-mail drjunmin@yahoo.com.cn (Jun MIN); cyj0509@126.com (Ya-jin CHEN)

Received 2010-03-16 Accepted 2010-06-23
}

Recent studies have shown that embryonic stem (ES) cells, with their unlimited proliferative ability and pluripotent differentiation potential, may provide an alternative cell source for replacement therapy in the treatment of various diseases ${ }^{[7]}$. A few studies have also indicated that ES cell-derived hepatocytes can secrete F-VIII or F-IX, suggesting features of coagulant activity and pointing to stem cell replacement as a potential therapy for hemophilia ${ }^{[8,9]}$. However, ES cell-derived hepatocytes that coexpress F-VIII and F-IX differentiate inefficiently. In addition, there are no comprehensive tests for F-VIII and F-IX production by these cells. Therefore, a stable, high-efficiency differentiation model is needed.

Sodium butyrate (SB) is a histone deacetylase inhibitor; it has been reported to promote the differentiation of many cell types via chromatin rearrangement and histone deacetylation $^{[10,11]}$. Our previous study ${ }^{[12]}$ and those of others ${ }^{[13-15]}$ have reported the use of SB in the high-efficiency induction of ES cells differentiation into hepatic progenitor cells and hepa- 
tocytes. However, whether these differentiated hepatocytes express F-VIII or F-IX is unknown.

Bone morphogenetic proteins (BMPs) and fibroblast growth factors (FGFs) have been shown to be required for the formation of endoderm cells ${ }^{[16,17]}$. Studies have shown that at the time of liver specification, the induction of hepatic fate requires $\mathrm{BMP} 4$ expression in the mesoderm surrounding the foregut endoderm ${ }^{[18-20]}$. Derived from the cardiac mesoderm, bFGF is required for the initiation of liver bud development in the anterior definitive endoderm ${ }^{[21,22]}$. Additional studies have indicated a synergistic effect between BMPs and FGFs in inducing hepatocyte differentiation ${ }^{[23]}$.

In our previous study, we developed a novel method to induce mouse ES cells to differentiate into hepatocytes by adding $\mathrm{SB}^{[12]}$. The aim of the present study was to adopt a modified approach to induce ES cells to differentiate into functional hepatocytes that expressed both F-VIII and F-IX. To achieve this goal, we combined bFGF, BMP4, and other maturation factors such as hepatocyte growth factor (HGF), oncostatin M (OSM), and dexamethasone (Dex $)^{[23-25]}$. We hoped to establish a simplified protocol for the derivation of hepatocytes expressing both F-VIII and F-IX, thereby providing a potential source of transplantable cells for cell replacement therapy in hemophilia.

\section{Materials and methods}

\section{Maintenance of embryonic stem cells}

Nondifferentiated E14 mouse ES cells (ATCC, Manassas, VA, USA) were maintained on gelatin-coated dishes in Dulbecco's modified Eagle's medium (DMEM; GIBCO, Grand Island, NY, USA) supplemented with 15\% fetal bovine serum (Hyclone, Rockville, MD, USA), $1000 \mathrm{U} / \mathrm{mL}$ recombinant mouse leukemia inhibitory factor (LIF; Chemicon, Temecula, CA, USA), $1 \%$ nonessential amino acids, $1 \mathrm{mmol} / \mathrm{L}$ glutamine, and 0.1 mmol/L $\beta$-mercaptoethanol (Sigma-Aldrich, St Louis, MO, USA). This culture medium, excluding LIF, was defined as the differentiation medium.

\section{Differentiation of embryonic stem cells}

Embryonic stem cells were trypsinized and cultured at a density of $5 \times 10^{5}$ cells $/ \mathrm{mL}$ in 6 -well plates coated with $0.1 \%$ gelatin. The cells were cultured in differentiation medium containing $2.5 \mathrm{mmol} / \mathrm{L} \mathrm{SB}$ (Sigma-Aldrich), $10 \mathrm{ng} / \mathrm{mL}$ bFGF (R\&D Systems, Minneapolis, MN, USA) and $10 \mathrm{ng} / \mathrm{mL}$ BMP4 (R\&D Systems). Six days after induction, the cells were grown in differentiation medium supplemented with $20 \mathrm{ng} / \mathrm{mL}$ HGF (R\&D Systems) for an additional 6 days. For the final 3 days of culture, cells were treated with $0.1 \mu \mathrm{mol} / \mathrm{L}$ Dex (SigmaAldrich) and $10 \mathrm{ng} / \mathrm{mL}$ OSM (R\&D Systems) (Figure 1). Spontaneously differentiated cells were used as controls.

Reverse-transcription polymerase chain reaction (RT-PCR) and real-time PCR

RT-PCR was performed to detect the gene expression of F-VIII and F-IX, as well as the expression of several endodermspecific markers (alpha fetoprotein [AFP], hepatocyte nuclear

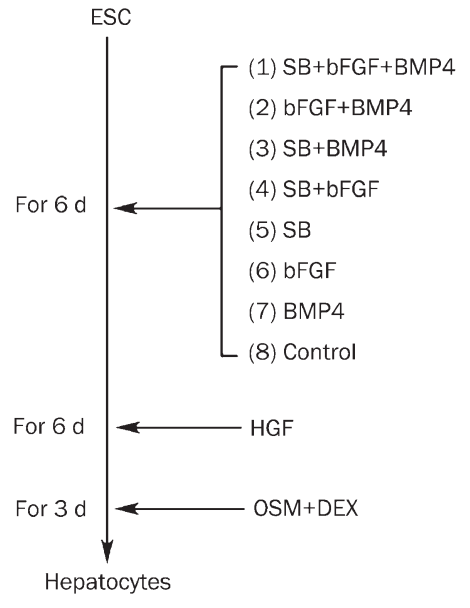

Figure 1. Schematic of the hepatocyte differentiation protocol.

factor $[\mathrm{HNF}]-3 \beta$, and $\mathrm{HNF}-4 \alpha)$, hepatocyte-specific markers (albumin [ALB], transthyretin [TTR], a1-antitrypsin [AAT], glucose-6-phosphate [G6P], cytokeratin 18 [CK18], cytokeratin-19 [CK19], and cytochrome P450 [CYP7a1]), and undifferentiated ES cells (Pou5f1). During various stages of the differentiation process, total RNA was extracted with TRIzol (Invitrogen, Carlsbad, CA, USA). A total of $2 \mu \mathrm{g}$ of RNA was used to prepare cDNA with the RevertAid First-Strand cDNA Synthesis Kit (Takara Bio Inc, Shiga, Japan). Samples of cDNA corresponding to the input RNA were amplified in PCR reaction buffer containing primers and LA Taq DNA polymerase (Takara Bio Inc). The PCR primer sequences are listed in Table 1. All quantifications were performed with the use of mouse $\beta$-actin as an internal standard. Semiquantitative analysis of PCR products was performed with an AlphaImager TM 2000 (Alpha Innotech, San Leandro, CA, USA), and the ratios of the markers to $\beta$-actin were calculated. Mouse hepatocytes were used as positive controls.

Real-time PCR was performed with a kit (Quantitect SYBR Green; Qiagen Inc) following the manufacturer's instructions. The total reaction volume was $25 \mu \mathrm{L}$, and $100 \mathrm{ng}$ of cDNA was used as the template. Fluorescence was detected using the Prism 7700 detection system (ABI, CA, USA). The relative quantification of gene expression was analyzed by the $2^{-\Delta \Delta C t}$ method, and the results were expressed as the extent of change with respect to the control values. Primer sequences used for real-time PCR are available on request.

\section{Electron microscopy}

On day 15 of cell differentiation, the culture plates were washed with phosphate-buffered saline (PBS) and fixed for $48 \mathrm{~h}$ in cold $25 \mathrm{~g} / \mathrm{L}$ glutaraldehyde in $0.1 \mathrm{~mol} / \mathrm{L}$ sodium cacodylate buffer ( $\mathrm{pH}$ 7.4). After fixation, the cells were harvested and centrifuged to form pellets. After postfixing in $10 \mathrm{~g} / \mathrm{L}$ osmium tetroxide in $0.1 \mathrm{~mol} / \mathrm{L}$ sodium cacodylate buffer $(\mathrm{pH}$ 7.4), the pellets were dehydrated in a graded alcohol series and embedded in low-viscosity epoxy resin. Ultrathin sections were stained with uranyl acetate and lead citrate and 
Table 1. Sequence information on the primers used for RT-PCR.

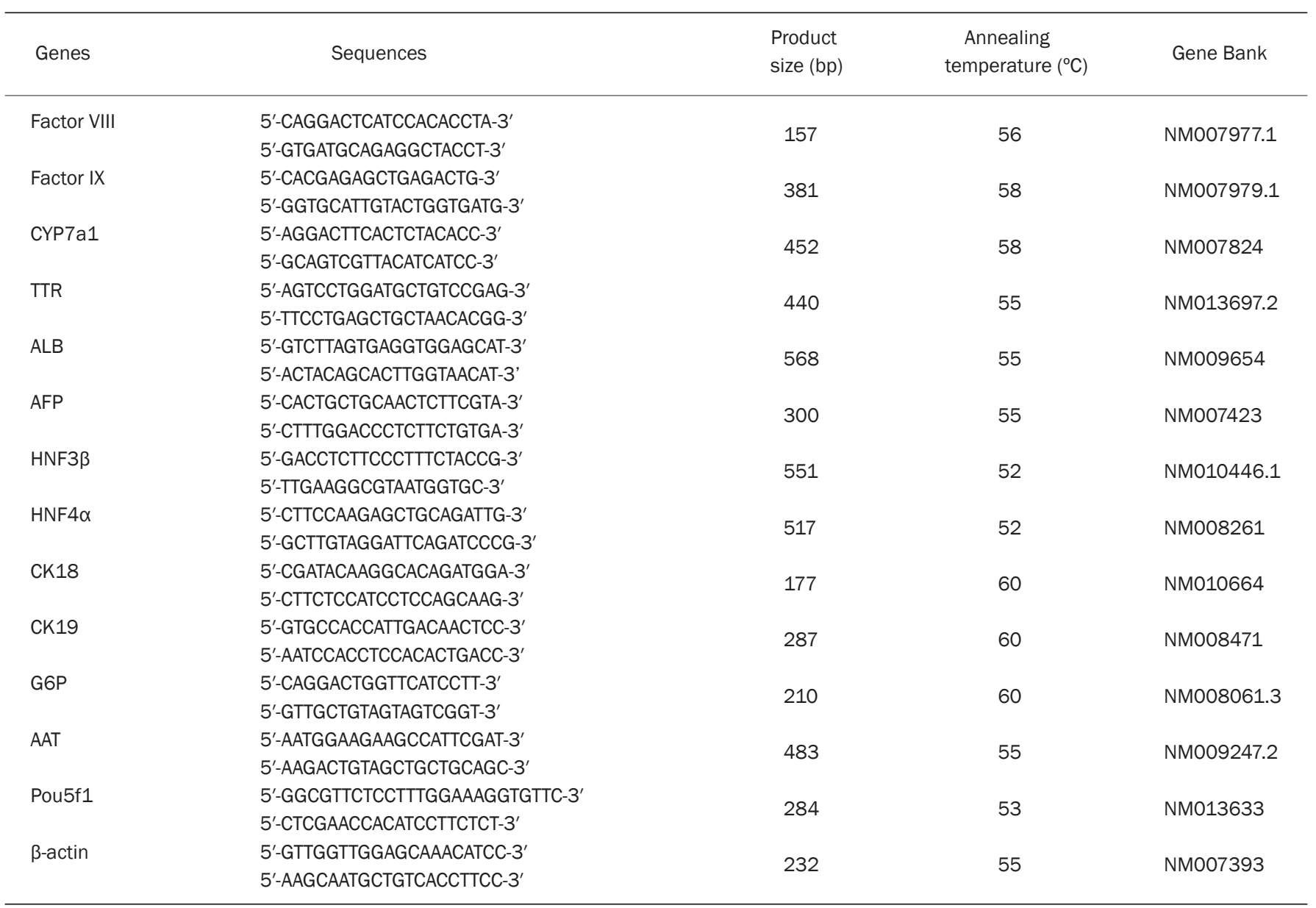

observed under an electron microscope.

\section{Immunocytochemistry}

On day 15 of cell differentiation, immunocytochemistry for F-VIII, F-IX, ALB, CYP7a1, and AFP was performed. The primary and secondary antibodies were obtained and diluted as follows: rabbit anti-mouse coagulation factor VIII heavy chain (1:100) and ALB (1:200) antibody (Santa Cruz Biotechnology, Santa Cruz, CA, USA) followed by Alexa Fluor 488-conjugated chicken anti-rabbit immunoglobulin G (IgG) (1:100, Molecular Probes, Eugene, OR, USA); goat anti-mouse coagulation factor IX (1:150), CYP7a1 (1:150) and AFP (1:200) antibody (Santa Cruz Biotechnology) followed by Alexa Fluor 555-conjugated donkey anti-goat IgG (1:100, Molecular Probes). Nuclear DNA was stained with 4',6-diamidino-2-phenylindole (DAPI) (Invitrogen). Undifferentiated ES cells were used as negative controls.

\section{Western blot analysis}

To detect F-VIII and F-IX proteins in ES cell-derived hepatocytes, Western blot analysis was performed. In brief, the cells were lysed in radioimmunoprecipitation assay (RIPA) buffer. A total of $10 \mu \mathrm{g}$ of protein was boiled, subjected to electropho- resis, and then transferred to a nitrocellulose membrane by semidry transfer. Membranes were blocked in Tris-buffered saline (TBS) containing 3\% nonfat milk and 0.05\% Tween 20 and then incubated in 1:1000 dilutions of rabbit anti-mouse coagulation factor VIII heavy chain antibody and goat antimouse coagulation factor IX antibody (Santa Cruz Biotechnology) at room temperature for $1 \mathrm{~h}$. The blots were rinsed and washed in TBS and then incubated in 1:10000 dilutions of horseradish peroxidase-conjugated secondary antibodies (donkey anti-goat IgG and chicken anti-rabbit IgG [heavy and light chains], Bio-Rad Laboratories, Hercules, CA, USA). The blots were developed with ECL Western blotting detection reagents (Amersham Biosciences UK Limited, England). Immunoreactive bands were quantified with an imaging system (AlphaImager 2000; Alpha Innotech Corp). Values were normalized to those of the internal control (glyceraldehyde 3-phosphate dehydrogenase [GAPDH]). Mouse hepatocytes were used as positive controls.

\section{Flow cytometry and ELISA}

In brief, cells at the final stage of differentiation were permeabilized with IntraPrep permeabilization reagent (Invitrogen) and then incubated with $0.2 \mu \mathrm{g}$ of rabbit anti-mouse coagula- 
tion factor VIII heavy chain antibody and goat anti-mouse coagulation factor IX antibody (Santa Cruz Biotechnology) and stained with the appropriate Alexa Fluor 488- and 555-conjugated secondary antibodies. Flow cytometry was performed with a fluorescence-activated cell sorter (Becton Dickinson, Franklin Lakes, NJ, USA) and CellQuest software (Becton Dickinson). Spontaneously differentiated cells were used as controls. ELISA (Affinity Biologicals, Ancaster, ON, Canada) was used to detect the levels of F-VIII and F-IX protein that were secreted into the culture medium following the manufacturer's instructions.

\section{Statistical analysis}

Each experiment was performed at least three times. All values are shown as means \pm standard deviation (SD). Data were analyzed with SPSS 10.0 software (SPSS Inc, Chicago, IL, USA). Statistical analysis was performed with one-way analysis of variance (ANOVA). Statistical significance was set at $P<0.05$.

\section{Results}

Differentiation of embryonic stem cells into hepatocyte-like cells During the process of differentiation, morphologic changes of ES cells were observed by light microscopy. On day 3 of induction with SB, the cells showed a polygonal shape that became more obvious by day 6 , while cells not treated with SB remained small and round. Morphologic differences became more pronounced by day 9. Most of these cells were epithelioid or fibroblast-like. This phenomenon indicates that SB promotes early morphologic changes in ES cells. On day 15 , cells exposed to SB+bFGF+BMP4 were the most homogeneous, hepatocyte-like cells compared to cells in other groups (Figure 2A). These cells were polygonal in shape with large, round, centered nuclei, and double nuclei were also present. Electron microscopy revealed that the differentiated cells were rich in endoplasmic reticulum, ribosomes, ellipsoids, and mitochondria, which are typical ultrastructural features of hepatocytes. A large amount of glycogen and the formation of glycogen pools could be seen, strongly indicating a secretory function (Figure 2B-2E). In the absence of any induction factors, control ES cells spontaneously differentiated into multiple lineages.

\section{Endoderm-specific and hepatocyte-specific markers are} expressed in differentiated cells

Gene expression for endoderm-specific markers (AFP, HNF-3 $\beta$, and HNF-4a) and hepatocyte-specific markers (ALB, TTR, AAT, G6P, CK18, CK19, and CYP7a1) was studied. Treatment with SB+bFGF+BMP4 induced higher levels of AFP, HNF-3 $\beta$, and HNF-4a expression. Moreover, at the final stage of differentiation (day 15), the definitive endoderm generated with $\mathrm{SB}+\mathrm{bFGF}+\mathrm{BMP} 4$ treatment showed the highest expression of mature hepatic markers such as CYP7a1, ALB, TTR, AAT, and G6P. The expression of Pou5f1, a marker of undifferentiated ES cells, gradually decreased (Figure 3A, 3B). Real-time PCR at day 15 revealed that cells treated with
SB+bFGF+BMP4 showed the highest expression of endodermspecific markers and hepatocyte-specific markers compared to other treatment groups (Figure 3C).

On day 15 of the differentiation protocol, immunocytochemistry of ES cell-derived hepatocytes showed costaining for ALB, AFP and CYP7a1 in cells treated with SB+bFGF+BMP4. The negative controls, undifferentiated ES cells, did not express ALB, AFP or CYP7a1 (Figure 3D).

These results indicate that the combination of SB+bFGF+ $\mathrm{BMP} 4$ is effective for generating cells that express endodermspecific and hepatocyte-specific markers.

The combination of sodium butyrate, basic fibroblast growth factor and bone morphogenetic protein 4 enhances mRNA expression of F-VIII and F-IX

We used RT-PCR and real-time PCR to analyze the gene expression of F-VIII and F-IX at various times during the culture process. In response to treatment with $\mathrm{SB}+\mathrm{bFGF}+\mathrm{BMP} 4$, F-VIII and F-IX were expressed by days 6 and 9, respectively. In contrast, F-VIII was not detected in other groups until day 9 , and F-IX was detected at a lower level on day 9 in the presence of SB+bFGF. By the end of the culture process (day 15), the expression of F-VIII and F-IX in cells treated with $\mathrm{SB}+\mathrm{bFGF}+\mathrm{BMP} 4$ was significantly higher than that of other groups (Figure $4 \mathrm{~A}-4 \mathrm{C}$ ).

The combination of sodium butyrate, basic fibroblast growth factor and bone morphogenetic protein 4 enhances protein expression of F-VIII and F-IX

To assess the protein expression of F-VIII and F-IX, we used immunocytochemistry. Double staining for F-VIII and F-IX was performed on day 15 in cells treated with $\mathrm{SB}+\mathrm{bFGF}+\mathrm{BMP} 4$. The negative control, undifferentiated ES cells, expressed neither F-VIII nor F-IX (Figure 4E).

Western blots also showed that on day 15, the protein expression of F-VIII and F-IX was significantly higher in cells treated with $\mathrm{SB}+\mathrm{bFGF}+\mathrm{BMP} 4$ than in the other groups (Figure 4F). In spontaneously differentiated cells, neither F-VIII nor F-IX could be detected at the protein level.

Flow cytometry was performed to quantify the percentage of F-VIII- and F-IX-double positive cells among the differentiated population. Results showed that $71.4 \%$ of cells treated with SB+bFGF+BMP4 coexpressed F-VIII and F-IX on day 15, which was greater than that of the other groups (Figure 4G).

ELISA was performed to determine the levels of F-VIII and F-IX protein secreted into the culture medium (Figure 4D). At the final stage of differentiation (day 15), F-VIII and F-IX protein could be secreted into the culture medium by ES cellderived hepatocytes treated with SB+bFGF+BMP4, which secreted the highest concentration compared to any other treatment groups.

\section{Discussion}

ES cell-derived hepatocytes capable of secreting F-VIII and F-IX may represent a unique source for the cell-based treatment of hemophilia. Our present results demonstrate that the 

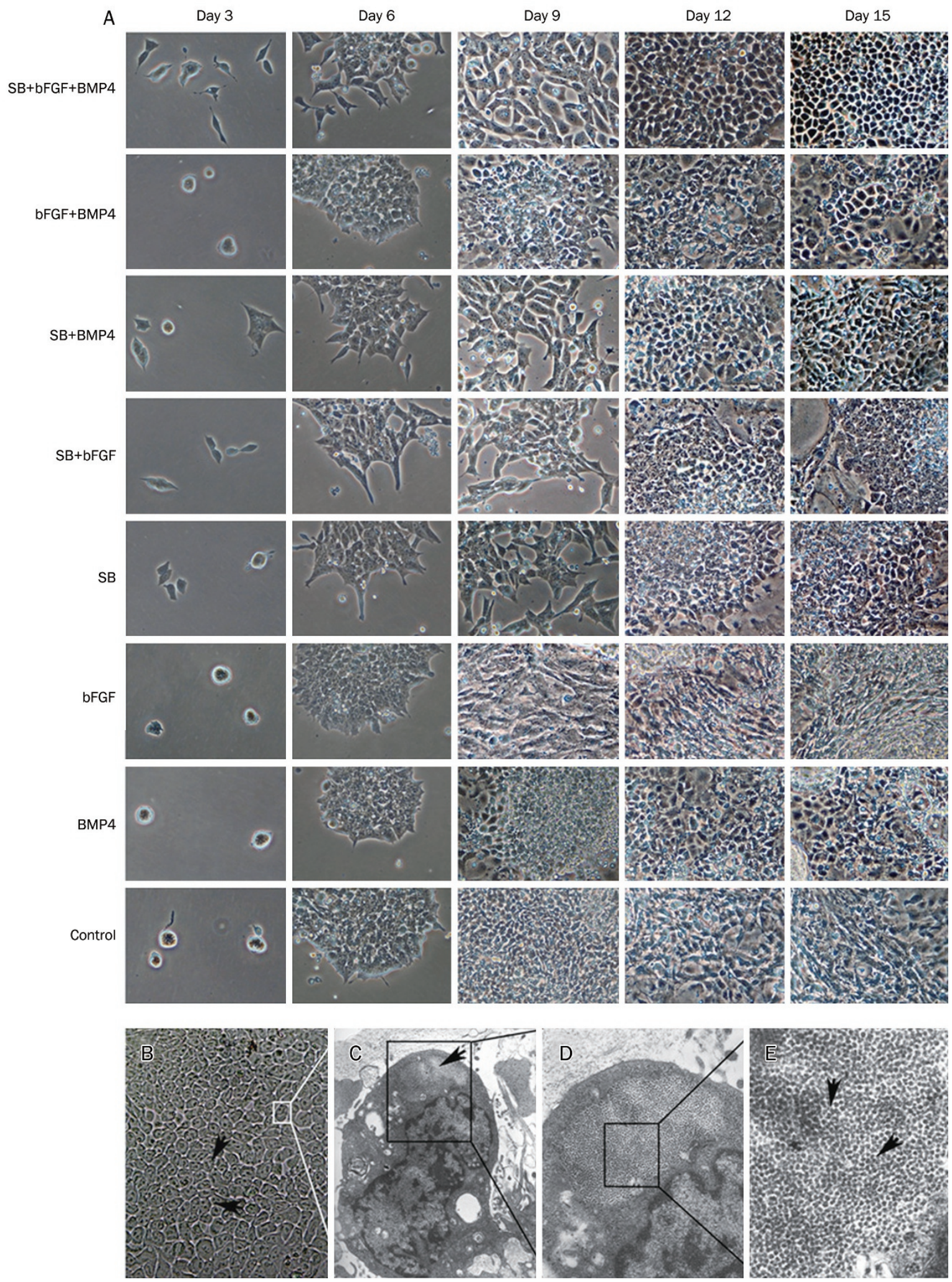

Figure 2. Changes in the morphology and ultrastructure of embryonic stem cells during the process of differentiation. (A) Light microscopic comparison of morphologic changes among cells treated with different combinations of sodium butyrate (SB), basic fibroblast growth factor (bFGF), and bone morphogenetic protein 4 (BMP4) in the early stages of differentiation. Spontaneously differentiated cells were used as controls (magnification, 200x). (B) Morphologic changes on day 15 in cells treated with SB, bFGF, and BMP4 seen through a light microscope (magnification, 400×). (C-E) Electron micrographs of day 15 cells treated with SB, bFGF, and BMP4 (magnification: [C] 10000×, [D] 20000×, [E] 50000×). Arrows in (C) and (E) indicate the formation of glycogen pools containing large amounts of glycogen. 
A

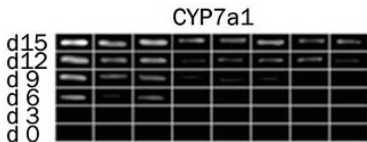

HNF-3 $\beta$

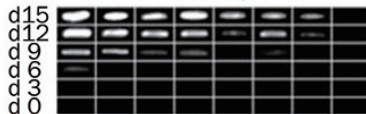

G6P
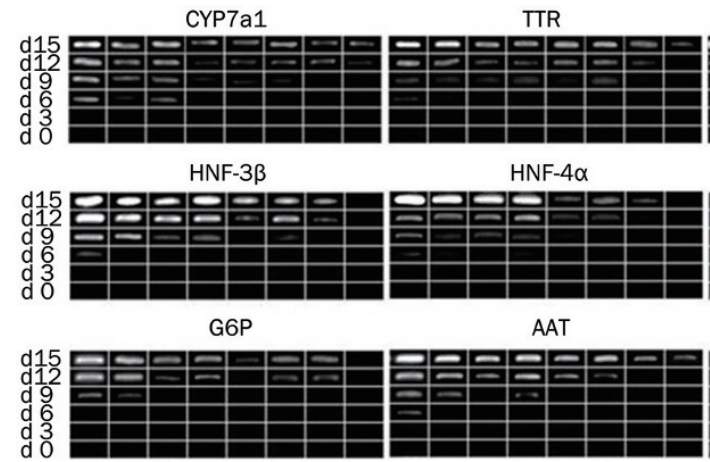

HNF-4 $\alpha$

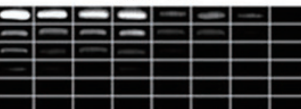

AAT

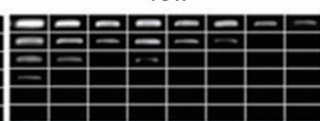

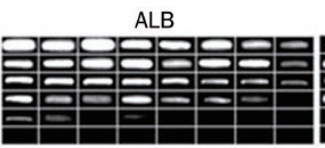

CK18

pou5f1

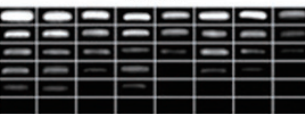

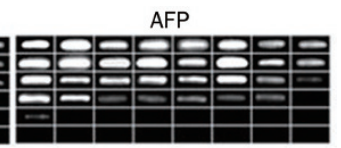

CK19

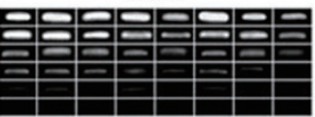

$\beta$-actin

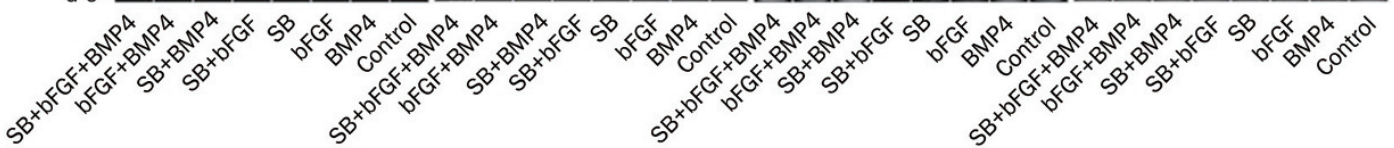

Mouse hepatocytes

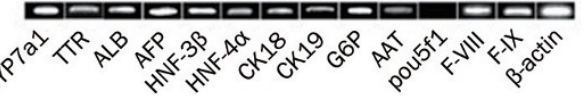
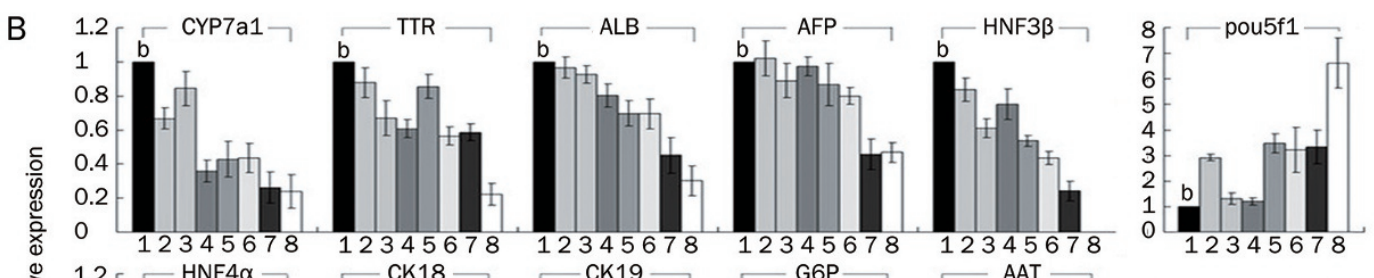

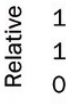
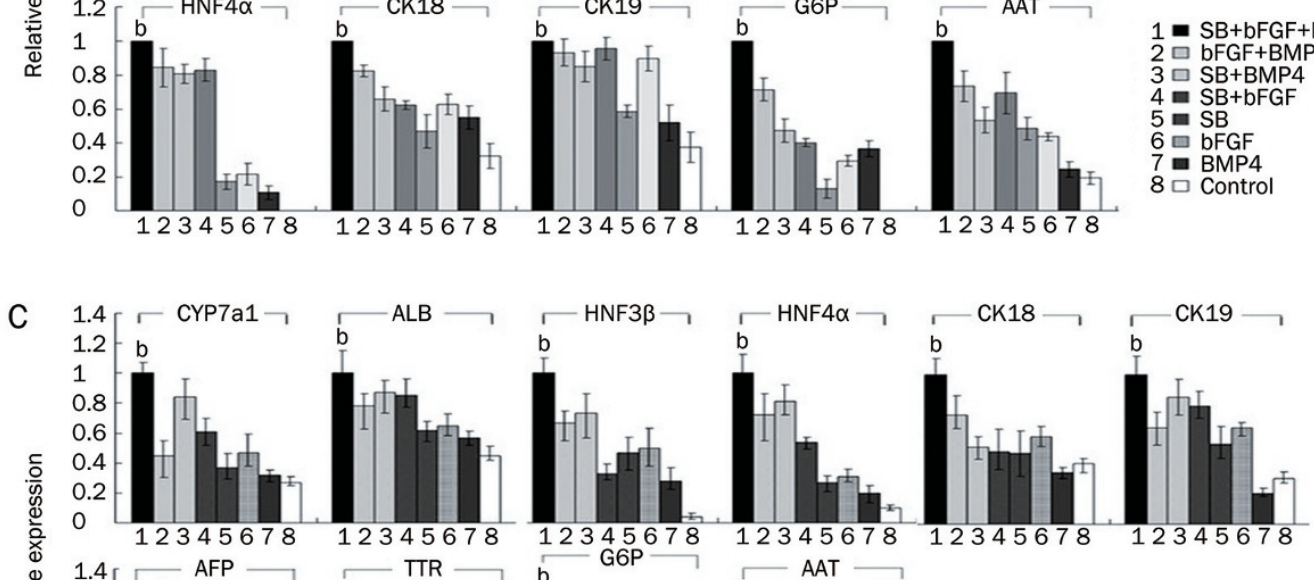

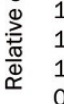
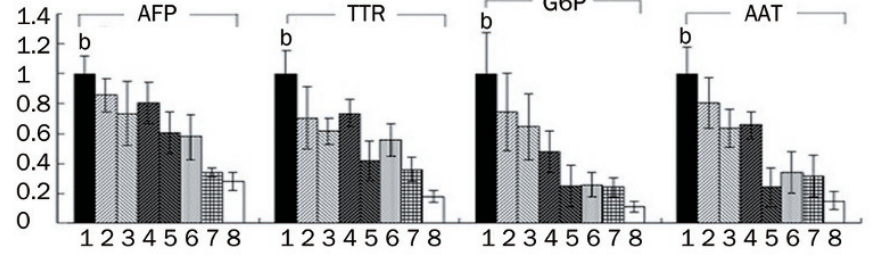

1 - SB+bFGF+BMP4

2 bFGF+BMP4

3 SB+BMP4

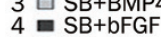

5 드

5
6 ㅁ bFGF

7 BMP4

8 Control
Figure 3. Expression of endoderm-specific and hepatocyte- specific markers in differentiated cells. (A) Reverse-transcription polymerase chain reaction (RTPCR) analysis of endodermspecific and hepato cytespecific markers during various stages of the differentiation process. Mouse hepatocytes were used as positive controls. (B) Comparison of gene expression among various cell groups on day 15 . The expression of $\beta$-actin mRNA was used as a loading control. Ratios of endodermspecific and hepatocytespecific markers to $\beta$-actin were calculated. Values are shown as means $\pm S D$. Histograms represent densitometric analysis corresponding to the mean $\pm S D$ of three independent experiments. ${ }^{\mathrm{b}} P<0.05$ vs any other group. (C) Real timePCR analysis of endodermspecific and hepatocytespecific markers at the final stage of differentiation (day 15). Histograms represent densitometric analysis corresponding to the mean $\pm S D$ of three independent experiments. ${ }^{b} P<0.05$ vs any other group. (D) Double immunofluorescence of albumin (ALB), alpha fetoprotein (AFP), and cytochrome P450 (CYP7a1) was detected on day 15 in cells differentiated with sodium butyrate (SB), basic fibroblast growth factor (bFGF), and bone morphogenetic protein 4 (BMP4). Cells were stained for ALB (green) and AFP (red) or CYP7a1 (red). Blue indicates nuclear staining with 4',6-diamidino-2-phenylindole (DAPI), and merged images are shown. Undifferentiated ES cells were used as negative controls. 

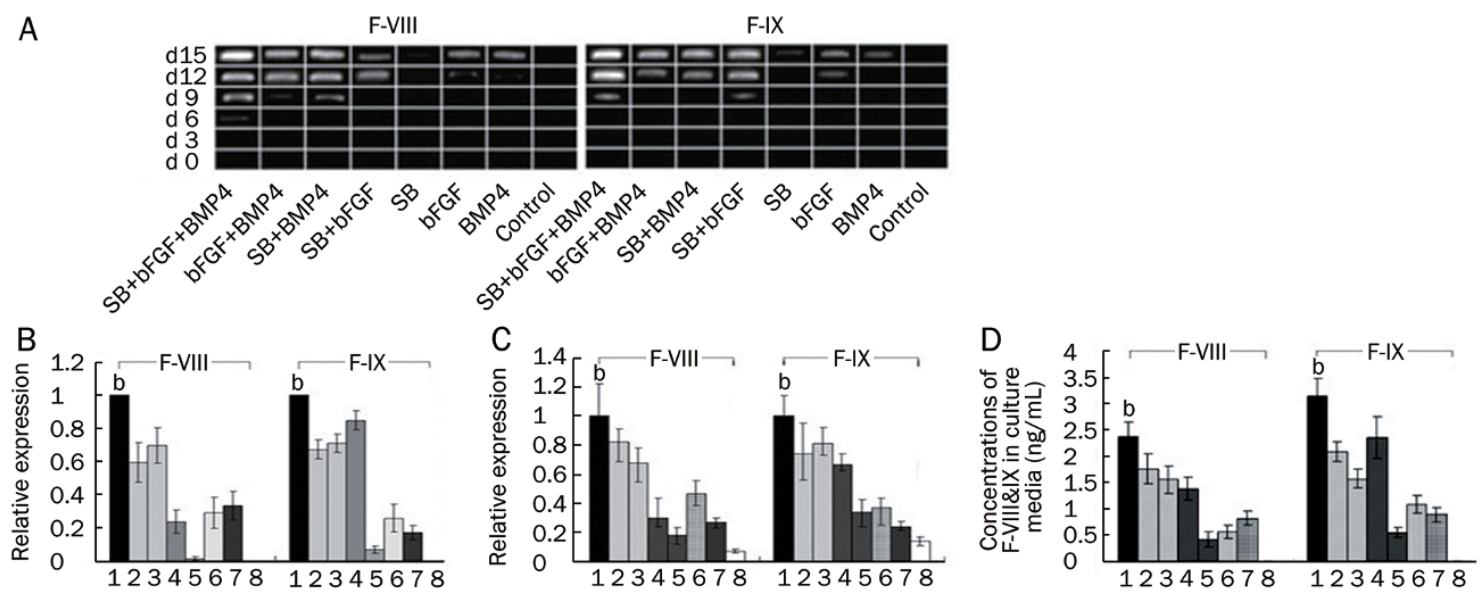

1 - SB+bFGF+BMP4

$2 \square \mathrm{bFGF+BMP}$

$3 \square$ SB+BMP4

4 B.bFGF

$5 \square \mathrm{SB}$

$6 \square \mathrm{bFGF}$

7 BMP4

7 BMP4

$8 \square$ Control

E
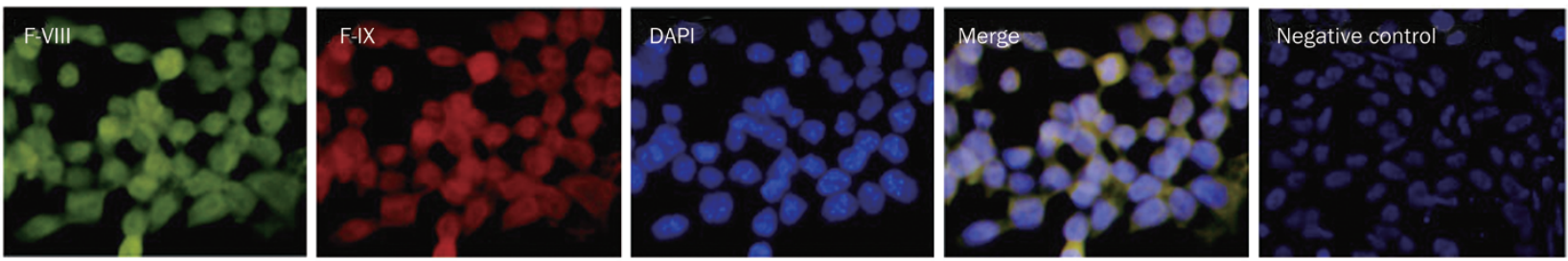

F
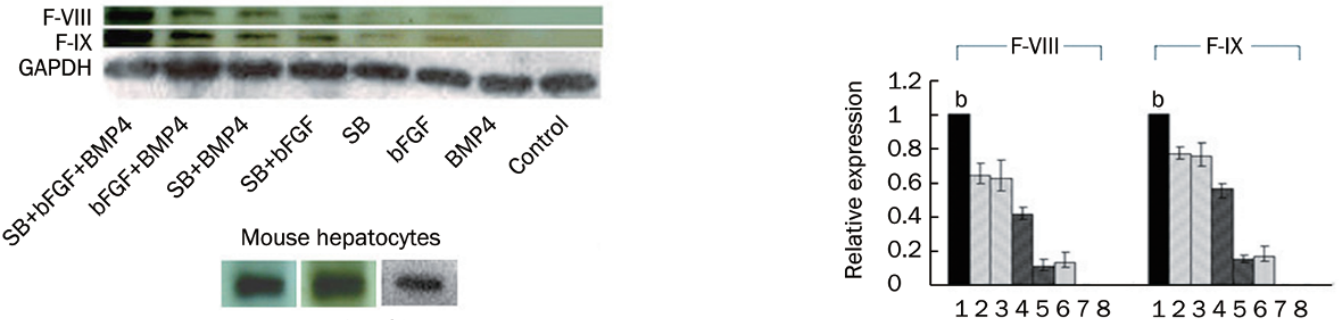

1 - SB+bFGF+BMP4

$2 \square \mathrm{bFGF+BMP4}$

$3 \square \mathrm{SB}+\mathrm{BMP} 4$

4 ․ SB+bFGF

$5=\mathrm{SB}$

$6 \square \mathrm{bFGF}$

7 BMP4

8 Control

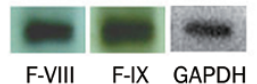

12345678

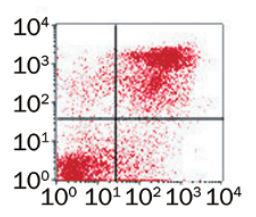

$\mathrm{SB}+\mathrm{bFGF}$

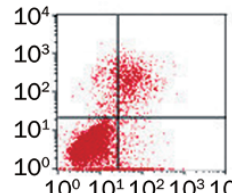

SB

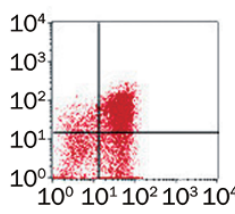

bFGF+BMP4

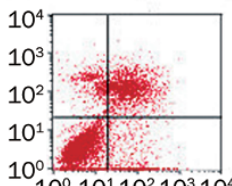

bFGF

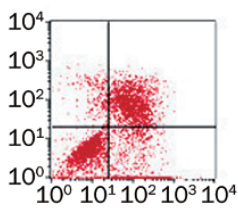

$\mathrm{SB}+\mathrm{BMP} 4$

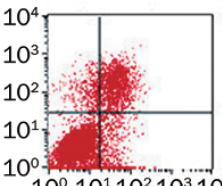

BMP4

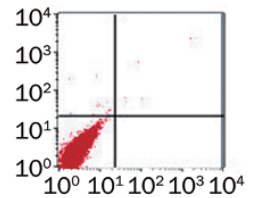

Control



1 - SB+bFGF+BMP4

$2 \square$ bFGF+BMP4

$3 \square \mathrm{SB}+\mathrm{BMP4}$

4 을 $+\mathrm{bFGF}$

5 드

$6 \square \mathrm{bFGF}$

7 BMP4

8 Control

Figure 4. Expression of F-VIII and F-IX in differentiated embryonic stem cells. (A) Reverse-transcription polymerase chain reaction (RT-PCR) analysis of $\mathrm{F}-\mathrm{VIII}$ and F-IX gene expression during various stages of the differentiation process. Comparisons among cells treated with different combinations of factors were performed. (B) Comparisons of F-VIII and F-IX gene expression among various cell groups on day 15 . The expression of $\beta$-actin mRNA was used as a loading control. Histograms represent densitometric analysis corresponding to the mean $\pm \mathrm{SD}$ of three independent experiments. ${ }^{b} P<0.05 \mathrm{vs}$ any other group. (C) Real time-PCR analysis of F-VIII and F-IX on day 15. Histograms represent densitometric analysis corresponding to the mean \pm SD of three independent experiments. ${ }^{b} P<0.05$ vs any other group. (D) ELISA results showing the protein levels of F-VIII and F-IX secreted into the culture medium. Histograms represent densitometric analysis corresponding to the mean $\pm S D$ of three independent experiments. ${ }^{b} P<0.05$ versus any other group. (E) Double immunofluorescence for F-VIII and F-IX was performed on day 15 in cells differentiated with SB+bFGF+BMP4. Cells were stained for F-VIII (green) and F-IX (red). Blue indicates nuclear staining with 4',6-diamidino-2-phenylindole (DAPI), and merged images are shown. Undifferentiated ES cells were used as negative controls. (F) Western blot of analysis of F-VIII and F-IX on day 15. Expression of glyceraldehyde 3-phosphate dehydrogenase (GAPDH) was used as a loading control. The histogram represents densitometric results from three independent experiments. ${ }^{\mathrm{b}} P<0.05$ vs any other group. Mouse hepatocytes were used as positive controls. (G) Flow cytometry experiments analyzed F-VIII and F-IX double-positive cells. The right upper corner represents F-VIII and F-IX double-positive cells. The histogram shows the percentage of cells coexpressing F-VIII and F-IX on day 15, and a comparison among groups treated with different combinations of factors was performed. All experiments were repeated three times. ${ }^{\mathrm{b}} \mathrm{P}<0.05$ vs any other group. 
combination of $\mathrm{SB}+\mathrm{bFGF}+\mathrm{BMP} 4$, when applied at the stage of hepatic progenitor differentiation, helps to prompt highefficiency differentiation of ES cells into hepatocytes and significantly enhances the mRNA and protein levels of F-VIII and F-IX. To our knowledge, this is the first report confirming the coexpression of F-VIII and F-IX in ES cells induced to differentiate into hepatocytes.

F-VIII and F-IX are secreted by mature hepatocytes, and the high-efficiency generation of mature hepatocytes from ES cells is a critical step. The differentiation of ES cells into hepatocytes is a promising approach for strategies aiming to mimic in vivo organogenesis. Our present protocol was designed to mimic hepatic development and included three developmental events: definitive endoderm formation, hepatic progenitor specification, and maturation. Our previous study and those of others have demonstrated that sodium butyrate, an inhibitor of histone deacetylase, induces the differentiation of ES cells into hepatocytes ${ }^{[12-15]}$. Some researchers have also shown that at the time of liver specification, BMP4 expression in the mesoderm surrounding the foregut endoderm is required for the induction of hepatic fate ${ }^{[18-20]}$ and that bFGF from the cardiac mesoderm is required for the initiation of liver bud development in the anterior definitive endoderm ${ }^{[21,22]}$. Zhou ${ }^{[23]}$ have reported a synergistic effect between bFGF and BMP4 in inducing hepatocyte differentiation. Soto-Gutierrez ${ }^{[25]}$ reported inducing ES cells to differentiate into hepatocytes by treating them with FGF-2, activin-A, and HGF and later by giving them OSM and Dex. All of these studies have indicated that the use of combinations of various inducing factors could significantly improve the differentiation efficiency of ES cellderived hepatocytes. However, the secretion of F-VIII and F-IX was not detected in these differentiated hepatocytes.

In the present study, we explored endoderm-specific and hepatocyte-specific gene expression in various groups of cells treated with different combinations of SB, bFGF, and BMP4 during hepatic progenitor differentiation. We found that the combination of SB+bFGF+BMP4 induced obvious morphologic changes in ES cells at an early stage of differentiation; nearly all of the endoderm-specific markers assayed (AFP, HNF-3 $\beta$, and HNF-4a) were expressed at significantly higher levels compared to those in cells treated with any two factors, and induction with a single factor did not result in highefficiency ES cell differentiation. More importantly, we demonstrated that the combination of SB+bFGF+BMP4, applied at an early stage of differentiation, can cause high expression of F-VIII and F-IX at the final stage of differentiation.

Sodium butyrate is thought to act in the differentiation of hepatocytes via histone deacetylation ${ }^{[10,11]}$. With respect to our present results, we speculate that the treatment of ES cells with SB triggered epigenetic changes, making these cells more responsive to bFGF- and BMP4-mediated endoderm-specific and hepatocyte-specific gene expression. However, exactly how these three factors synergize and interrelate in the hepatic differentiation of ES cells is still undefined and remains to be elucidated.
To verify the potential of these hepatic progenitor cells to differentiate into F-VIII- and F-IX-secreting hepatocytes, we used a combination of exogenous inducing factors in our differentiation protocol. We found that the later addition of HGF, OSM, and Dex induced even more differentiated hepatocyte-like cells at the final stage of differentiation, which was confirmed by the increased expression of mature hepatocyte markers such as ALB and CYP7A1. In addition, double staining for ALB, CYP7a1, and AFP proteins, as well as the formation of glycogen pools in cells treated with SB+bFGF+BMP4, suggests a function similar to that of mature hepatocytes. This result demonstrates that the combination of SB, bFGF, and $\mathrm{BMP} 4$ at the stage of hepatic progenitor differentiation leads to effective hepatocyte differentiation. Furthermore, we assessed F-VIII and F-IX expression in response to different combinations of factors. Our present results show that treatment with $\mathrm{SB}+\mathrm{bFGF}+\mathrm{BMP} 4$ at the stage of hepatic progenitor differentiation enhances the mRNA and protein expression of both F-VIII and F-IX. This treatment also resulted in an increase in the percentage of cells coexpressing F-VIII and F-IX. Additionally, the levels of F-VIII and F-IX protein that were secreted into the culture medium increased. We speculate that SB exerts a synergistic effect with bFGF and BMP4 in promoting the differentiation of ES cell-derived hepatocytes that express both F-VIII and F-IX.

The absence of a hepatocyte phenotype during the in vitro induction of hepatocytes from ES cells is a problem. It has been reported that ES cell-derived hepatocytes are capable of secreting either F-VIII or F-IX protein ${ }^{[8,9]}$, However, the coexpression of F-VIII and F-IX from ES cell-derived hepatocytes has remained inefficient. Our present results show that both F-VIII and F-IX are stably expressed in ES cell-derived hepatocytes, and this phenotype is closer to that of mature hepatocytes. The production of ES cell-derived hepatocytes coexpressing both F-VIII and F-IX may provide a valuable replacement therapy for patients suffering from combined F-VIII and F-IX deficiency.

In conclusion, our present results demonstrate that at the early stage of hepatic progenitor formation, the combination of SB, bFGF, and BMP4 promotes ES cells to differentiate into hepatocytes that secrete both F-VIII and F-IX with high efficiency. This study provides a foundation for stem-cell replacement therapy for hemophilia A and B. Future studies are required to elucidate the signaling pathways underlying ES cell-derived hepatocyte differentiation.

\section{Acknowledgements}

This work was supported by grants from the China National Programs for High Technology Research and Development (No 2007AA02Z117), the National Natural Science Foundation of China (No 30571805 and 30672036), the Science and Technology project of Guang Dong province (№ 2008B080703028), the Doctoral Fund of Youth Scholars of Ministry of Education of China (№ 200805581132), and the China Postdoctoral Special Foundation. 


\section{Author contribution}

Jun MIN and Ya-jin CHEN designed research; Jun CAO and Chang-zhen SHANG performed research; Li-hong LÜ and De-chuan QIU contributed new analytical tools and reagents; Meng REN analyzed data; Jun CAO wrote the paper.

\section{References}

1 Saenko EL, Ananyeva N, Kouiavskaia D, Schwinn H, Josic D, Shima M, et al. Molecular defects in coagulation Factor VIII and their impact on Factor VIII function. Vox Sang 2002; 83: 89-96.

2 Castaldo G, Nardiello P, Bellitti F, Santamaria R, Rocino A, Coppola A, et al. Haemophilia B: from molecular diagnosis to gene therapy. Clin Chem Lab Med 2003; 41: 445-51.

3 Bowen DJ. Haemophilia A and haemophilia B: molecular insights. Mol Pathol 2002; 55: 127-44.

4 Engelfriet $\mathrm{CP}$ RH. Prophylactic treatment of patients with haemophilia A or haemophilia B. Vox Sang 2005; 88: 62-7.

5 Bolton-Maggs $\mathrm{PH}$. Optimal haemophilia care versus the reality. $\mathrm{Br} \mathrm{J}$ Haematol 2006; 132: 671-82.

6 Ohashi K, Waugh JM, Dake MD, Yokoyama T, Kuge H, Nakajima Y, et al. Liver tissue engineering at extrahepatic sites in mice as a potential new therapy for genetic liver diseases. Hepatology 2005; 41: 132-40.

7 Prelle K, Zink N, Wolf E. Pluripotent stem cells - model of embryonic development, tool for gene targeting, and basis of cell therapy. Anat Histol Embryol 2002; 31: 169-86.

8 Kasuda S, Kubo A, Sakurai Y, Irion S, Ohashi K, Tatsumi K, et al. Establishment of embryonic stem cells secreting human factor VIII for cell-based treatment of hemophilia A. J Thromb Haemost 2008; 6: 1352-9.

9 Fair JH, Cairns BA, Lapaglia MA, Caballero M, Pleasant WA, Hatada $\mathrm{S}$, et al. Correction of factor IX deficiency in mice by embryonic stem cells differentiated in vitro. Proc Natl Acad Sci USA 2005; 102: 2958-63.

10 Blouin MJ, Lamy I, Loranger A, Noel M, Corlu A, Guguen-Guillouzo $C$, et al. Specialization switch in differentiating embryonic rat liver progenitor cells in response to sodium butyrate. Exp Cell Res 1995; 217: 22-30.

11 McKiernan E, O'Driscoll L, Kasper M, Barron N, O'Sullivan F, Clynes M. Directed differentiation of mouse embryonic stem cells into pancreatic-like or neuronal- and glial-like phenotypes. Tissue Eng 2007; 13: 2419-30.

12 Min J, Shang CZ, Chen YJ, Zhang L, Liu L, Deng XG, et al. Selective enrichment of hepatocytes from mouse embryonic stem cells with a culture system containing cholestatic serum. Acta Pharmacol Sin
2007; 28: 1931-7.

13 Rambhatla L, Chiu CP, Kundu P, Peng Y, Carpenter MK. Generation of hepatocyte-like cells from human embryonic stem cells. Cell Transplant 2003; 12: 1-11.

14 Sharma NS, Shikhanovich R, Schloss R, Yarmush ML. Sodium butyrate-treated embryonic stem cells yield hepatocyte-like cells expressing a glycolytic phenotype. Biotechnol Bioeng 2006; 94: 1053-63.

15 Zhou QJ, Xiang LX, Shao JZ, Hu RZ, Lu YL, Yao H, et al. In vitro differentiation of hepatic progenitor cells from mouse embryonic stem cells induced by sodium butyrate. J Cell Biochem 2007; 100: 29-42.

16 Czyz J, Wobus A. Embryonic stem cell differentiation: the role of extracellular factors. Differentiation 2001; 68: 167-74.

17 Schuldiner M, Yanuka O, Itskovitz-Eldor J, Melton DA, Benvenisty N. Effects of eight growth factors on the differentiation of cells derived from human embryonic stem cells. Proc Natl Acad Sci USA 2000; 97 : 11307-12.

18 Gouon-Evans V, Boussemart L, Gadue P, Nierhoff D, Koehler Cl, Kubo A, et al. BMP-4 is required for hepatic specification of mouse embryonic stem cell-derived definitive endoderm. Nat Biotechnol 2006; 24: 1402-11.

19 Rossi JM, Dunn NR, Hogan BL, Zaret KS. Distinct mesodermal signals, including BMPs from the septum transversum mesenchyme, are required in combination for hepatogenesis from the endoderm. Genes Dev 2001; 15: 1998-2009.

20 Gong SG, Guo C. Bmp4 gene is expressed at the putative site of fusion in the midfacial region. Differentiation 2003; 71: 228-36.

21 Jung J, Zheng M, Goldfarb M, Zaret KS. Initiation of mammalian liver development from endoderm by fibroblast growth factors. Science 1999; 284: 1998-2003.

22 Ishikawa T, Terai S, Urata Y, Marumoto Y, Aoyama K, Sakaida I, et al. Fibroblast growth factor 2 facilitates the differentiation of transplanted bone marrow cells into hepatocytes. Cell Tissue Res 2006; 323: 221-31.

23 Zhou QJ, Huang YD, Xiang LX, Shao JZ, Zhou GS, Yao H, et al. In vitro differentiation of embryonic stem cells into hepatocytes induced by fibroblast growth factors and bone morphological protein-4. Int J Biochem Cell Biol 2007; 39: 1714-21.

24 Ong SY, Dai H, Leong KW. Hepatic differentiation potential of commercially available human mesenchymal stem cells. Tissue Eng 2006; 12: 3477-85.

25 Soto-Gutierrez A, Kobayashi N, Rivas-Carrillo JD, Navarro-Alvarez N, Zhao D, Okitsu T, et al. Reversal of mouse hepatic failure using an implanted liver-assist device containing ES cell-derived hepatocytes. Nat Biotechnol 2006; 24: 1412-9. 